\title{
Effect of Streamwise Vortices on Wake Properties Associated with Sound Generation
}

\author{
ARNOLD M. KUETHE* \\ The University of Michigan, Ann Arbor, Mich.
}

\begin{abstract}
Experiments in the turbulent-boundary-layer range of Reynolds numbers show that the Kármán vortex street is suppressed and spanwise periodicity is induced in the wake behind an airfoil by the introduction of inclined surface waviness near the trailing edge. Both effects will reduce the intensity of sound sources in many applications.
\end{abstract}

\section{Introduction}

$\mathbf{T}$ WO important aspects of the viscous wake leading to the generation of sound sources are: 1) unsteady twodimensional disturbances such as the Kármán vortex street, and 2) unsteady forces generated by the passage of a rotor blade through the two-dimensional wake of a stator, or vice versa. The unsteady wake of an aircraft makes a significant contribution to the total noise during the landing approach, whereas the unsteady forces due to wake impingement on stator and rotor blades contribute significantly during takeoff and cruise. The latter effect, analyzed by Kemp and Sears, ${ }^{1}$ is responsible for about half of the pure-tone noise generated by turbomachines; the other half originates with the mutual interference between the rotor and stator flowfields. ${ }^{2}$

Wind-tunnel tests reported here show that the streamwise vortices shed from vortex generators near the trailing edges of a flat plate and an airfoil have a strong suppression effect on the formation of the Kármán vortex street, and reduce the "effective" area of the velocity deficit in the wake, as seen by the following blade in a turbomachine. Both effects tend to suppress significantly the formation of sound sources.

\section{Vortex Generator}

The vortex generators used in these tests consisted of "wave elements,"3 of semi-circular, cross section, attached to the surfaces near the trailing edges and inclined to the external flow at an angle of about $15^{\circ}$. The flow over a wave element is shown schematically in Fig. 1. The element, which lies within the boundary layer, is inclined so as to deflect the vorticity vector of the boundary-layer flow toward the downstream direction, thus forming streamwise vortices in the layer very near the surface. The wake behind the element possesses vorticity of its own but the element also provides the means for decreasing the boundary-layer thickness by diverting vorticity into streamwise vortices, which have the familiar effect of enhancing the momentum transfer to the flow near the surface. Other vortex generators such as the vane and ramp types incorporate this feature ${ }^{4,5}$ but they depend primarily on deflecting momentum from the external flow toward the surface and are not designed to gather vorticity from the boundary layer over the entire spanwise extent of their utilization.

The major parameters for the design of an optimum configuration of wave-type generators are the height, width, and

Received March 21, 1972; revision received June 26, 1972. This investigation was supported by the Battelle Development Corporation. D. S. Brewer, F. S. Nelson, and C. Iott assisted with the experiments.

Index categories: Aerodynamic and Power Plant Noise; Jets, Wakes, and Viscid-Inviscid Flow Interactions; Nonsteady Aerodynamics.

* Professor, Aerospace Engineering. Fellow AIAA.

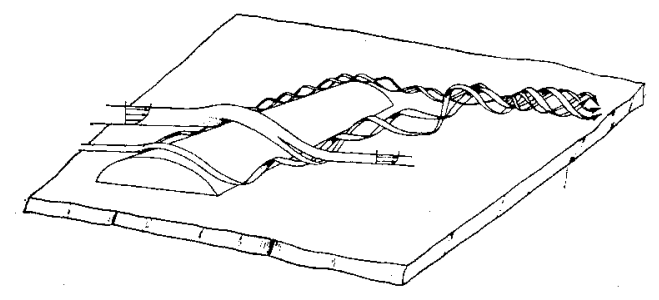

Fig. 1 Schematic flow over surface wave element in boundary layer.

length of the wave elements relative to the boundary layer thickness, their inclination to the flow, and their location on a surface. The elements could be in the form of either, or both, crests or troughs.

Comparison of various configurations of wave-type generators with a conventional vane-type configuration was carried out by Luce and Hoehne. ${ }^{6}$ The tests, at a Reynolds number of $10^{5}$, on an NACA63-012 airfoil, indicate that the wave system is competitive with the vane system in its effect to increase maximum lift with minimum drag penalty.

The diversion and concentration of boundary-layervorticity into secondary flows is a familiar phenomenon in turbomachines. ${ }^{7,8}$ In some instances iarge secondary circulation patterns develop, leading to serious flow distortions and losses in efficiency. If an appreciable portion of the vorticity in the boundary layers can be diverted into relatively small scale counter-rotating streamwise vortices, the net vorticity within any substantial region of the flow will be small and the intense lateral mixing associated with the vortices will tend to counteract the formation of large scale nonuniformities.

The experiments described below indicate that significant gains in this direction are possible.

\section{Results and Discussion}

Three exploratory experimental investigations were carried out in the wind tunnels at The University of Michigan. They were: 1) vorticity measurements to identify the vortex configuration and strength in the wake, 2) wake velocity traverses behind an airfoil showing the distortion of the wake by streamwise vortices, and 3) schlieren flash photographs to show the effect of vortex generators in suppressing the Kármán vortex street.

The vortex generators, mounted near the trailing edges of a plate and an airfoil, consisted of parallel elements placed at such a distance apart that counter-rotating vortices are generated. The process of formation can be described with reference to Figs. 1 and 2 . The vortices generated by the parallel elements themselves would be corotating as in Fig. 2a. If they are moved closer to each other a vortex sheet will develop; if, however, they are moved farther apart the corotating system diverts boundary-layer vorticity to form 


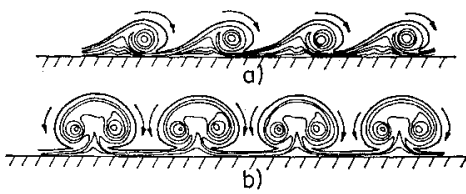

Fig. 2 Schematic cross section of streamwise a) co-rotating and b) counter-rotating vortices behind a surface with vortex generators.

secondary vortices of opposite sense in the intervening spaces, thus converting the flow to the counter-rotating system shown in Fig. $2 b$.

This flow configuration is shown by measurements of the vorticity in the wake of an airfoil. The airfoil thickness distribution was approximately that of the Clark Y with about $4 \%$ camber. It was rectangular, 12.5-in. chord by 24-in. span, with end plates of 18 in. diameter, with vortex generators on the suction side only. The lift coefficient was 0.4. Vorticity measurements were made in the wake by means of a McCormick rotating vane vorticity meter, with a rotor diameter of $.375 \mathrm{in}$. The tests were carried out in the $5 \mathrm{ft} \times 7 \mathrm{ft}$ wind tunnel at a speed of $110 \mathrm{ft} / \sec \left(R e=7 \times 10^{5}\right)$.

The measured distribution of angular velocity, $\omega$, in radians per second, in the wake is plotted in Fig. 3. The dimensions of the vortex generator array are also given, along with the location of the traverse station.

The measurements show the array of primary and secondary vortices of opposite senses described preceding. They are of roughly equal strength, each about $.375 \mathrm{in}$. in diameter, half the distance between the vortex generators. Since the diameter of the rotor of the vorticity meter was also 375 in, the sawtooth distribution shown in Fig. 3 represents the angular velocity of the rotor caused by the vorticity distribution across the streamtube of diameter .375 in. If the fluid within the streamtube is in solid body rotation the vorticity is constant and equal to that indicated by the vorticity meter with its axis at the center; for other vorticity distributions the measured angular velocity at the center indicates only some kind of average vorticity over the tube cross section.

The effect of the streamwise vortices on the mean velocity distribution in the wake was determined by means of spanwise hot wire traverses just aft of the airfoil trailing edge. The measurements were made on the wing used for the results

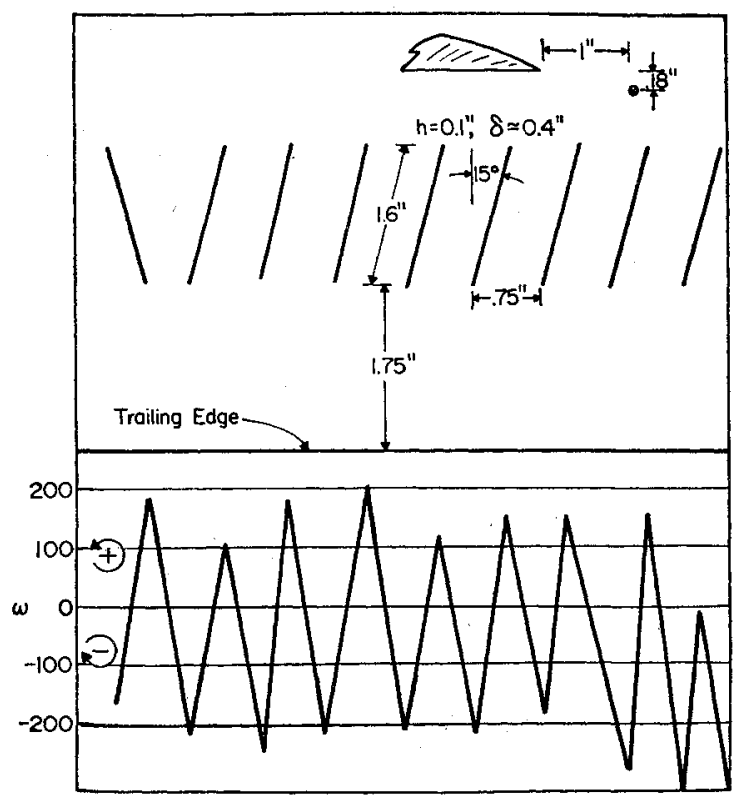

Fig. 3 Measured angular velocities in .375-in. diam. stream-tubes behind an airfoil with vortex generators.
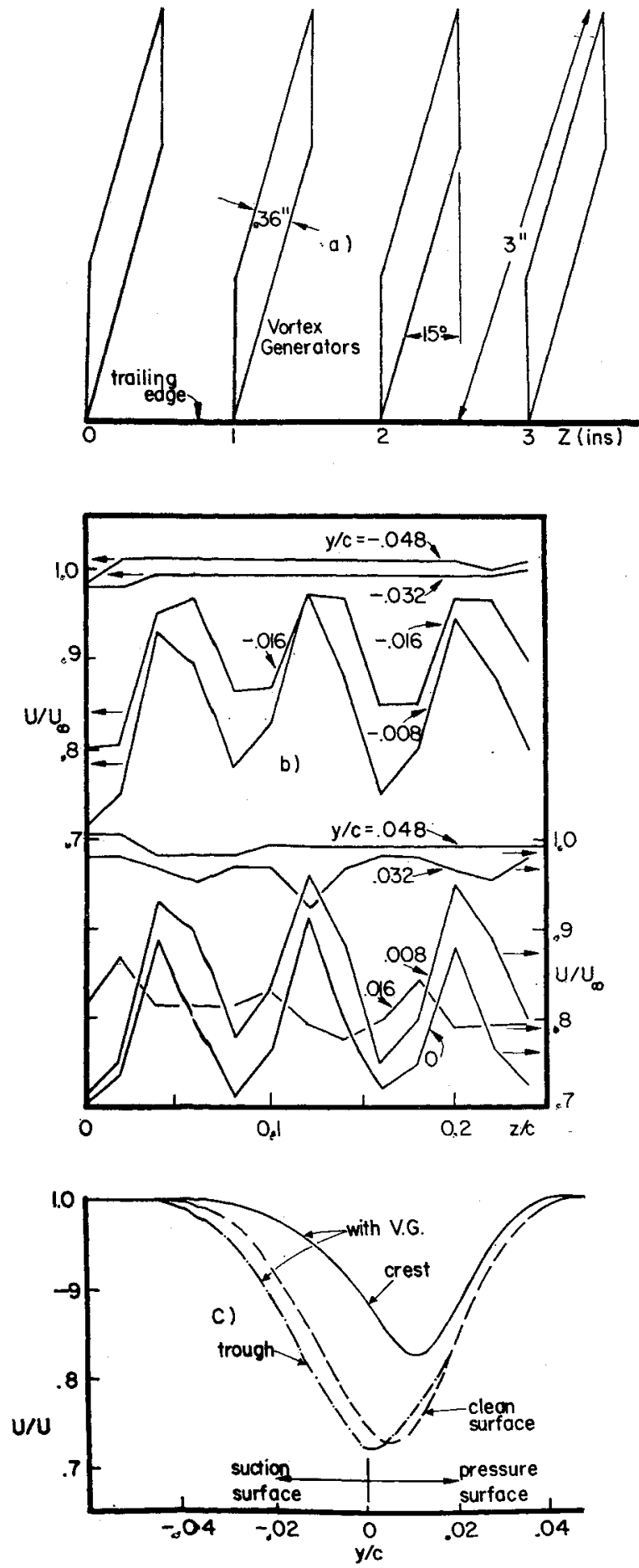

Fig. 4 Mean velocity measurements in the wake 2 in. downstream of the trailing edge of an airfoil with and without vortex generators on the suction surface; a) planview of vortex generators (.18 in. high); b) spanwise $(z / c)$ traverses at various $y / c$ (ordinate is shifted .3 , as indicated, for $\pm y / c$ traverses); c) wake profiles derived from spanwise traverses. $\quad c_{1}=0.4, \operatorname{Re}=3.5 \times 10^{5}$.

shown in Fig. 3 at a Reynolds number of $3.5 \times 10^{5}$ in a $2 \mathrm{ft} \times 2 \mathrm{ft}$ low turbulence tunnel at a lift coefficient of 0.4 Because of the low Reynolds number, masking tape was used to trip the boundary layer at about the $60 \%$ chord point. The boundary layer traverses 1 in. upstream of the trailing edge on the clean airfoil showed turbulent boundary layers .45 in. and .35 in. thick, respectively, on the suction and pressure surfaces.

The plan view of the configuration of vortex generators on the suction surface of the airfoil is shown in Fig. 4a. The 
generators were .18 in. high, of semi-circular, cross section except for the fore and aft regions, which were faired as shown; in the aft region the generators were faired in elevation as well. In addition the lee surfaces were faired smoothly into the airfoil surface with a small radius fillet of modeling clay.

Figure $4 \mathrm{~b}$ shows traverses of relative mean velocity along various spanwise paths at a distance of $2 \mathrm{in} .(x / c=0.16) \mathrm{aft}$ of the trailing edge. The origin for $Z$, the spanwise coordinate, is as shown at the trailing edge location of the first vortex generator. The $y$ coordinate was normal to and measured from the plane of the pressure surface of the airfoil. The lengths are nondimensionalized with the airfoil chord, $12.5 \mathrm{in}$. The origin for $U / U_{\infty}$ for the positive and negative $y / c$ traverses was displaced 0.3 as indicated by the arrows. The spanwise periodicities exhibited by the wake traverses are generated by the flowfields of the streamwise vortices shed by the inclined surface waviness; the traces are seen to be in phase over almost the entire wake.

In Fig. $4 c$ the mean crest and trough velocity distributions across the wake are plotted along with that for the clean surface.

The effect of vortex generators on the unsteady lift experienced by a following blade cutting through the wake will depend on its response to the spanwise periodic angle of attack variation. If the lift at each spanwise location responded to the velocity distribution at that station without attenuation due to spanwise gradients, the mean spanwise unsteady lift would be midway between the two extremes. However, a following blade sees the common region, that is, the crest distribution, as a two-dimensional wake and the spanwise periodic excursions to the trough distributions as superimposed perturbations. Since the scale of these perturbations is only about 0.1 chord their effect on the unsteady lift would be quite small and, therefore, the lift response of the blade would be near that for the crest distribution. The analysis by Kemp and Sears ${ }^{1}$ shows that the unsteady lift of a blade cutting through a wake is approximately proportional to the drag of the wake-producing blade; thus, the relative areas of the effective wakes with and without the vortex generators indicates the approximate unsteady lift reduction due to the vortex generators. The wake areas were compared and the results of Fig. 4 indicate a $50 \%$ reduction in the amplitude of the lift fluctuations for the crest distribution. Table 1 gives further results, in the form of the ratio of the wake area with vortex generators to that for the clean surface $\left(A_{v g} / A_{\mathrm{cl}}\right)$ at $1 \mathrm{in}$. and $2 \mathrm{in}$. behind the trailing edge $(x / c)$, with vortex generators of two heights $(h / \delta)$ on both surfaces, and on the suction surface only. For the last two tests small radius clay fillets were applied at the lee juncture between the surface and the vortex generators. The experimental results indicate that the use of vortex generators near the trailing edge on either or both surfaces can result in a substantial attenuation of the unsteady force resulting from wake impingement on a following surface. The attenuation is greater at $x / c=.16$ than at .08 ; considering that the phenomenon is associated with streamwise vortices that will disperse in the turbulent

Table 1 Ratio of area of velocity deficit in wake for crest distribution with vortex generators $\left(A_{v g}\right)$ to that for the clean surface $\left(A_{\mathrm{c} 1}\right)$ for two generator heights relative to boundary-layer thickness $(h / \delta)$, and two distances behind trailing edge in chords $(x / c)$, on both surfaces and on suction surface only

\begin{tabular}{cclc}
\hline \hline$x / c$ & $h / \delta$ & Surfaces & $A_{\text {vg }} / A_{\mathrm{cl}}$ \\
\hline .08 & .27 & both & .70 \\
.08 & .27 & suction & .77 \\
.16 & .27 & both & .53 \\
.16 & .27 & suction & .60 \\
.08 & .42 & both & .58 \\
.08 & .42 & suction & .86 \\
.16 & .42 & both & .60 \\
.16 & .42 & suction & .50 \\
\hline \hline
\end{tabular}

wake, there is probably a value of $x / c$ at which the attenuation is a maximum.

Another method for attenuating the unsteady forces due to wake impingement is that of tilted vanes described by Rao. ${ }^{9}$ Large decreases in sound level were measured for various tilted vane configurations in which the following blade intersects the wake obliquely. Tilted vanes and the use of vortex generators are thus different methods for introducing three dimensionality into the wake as seen by a following blade. The acoustic measurements by Rao indicate that such manipulations of the wake can reduce significantly the sound level generated by the fluctuating forces.

Another feature of the generation of counter-rotating vortex systems is that the net vorticity over a given volume would be small, so that the development of large-scale nonuniformities will be inhibited. That this effect can be significant is indicated by an eddy viscosity associated with the vortices.

The magnitude of the eddy viscosity $\varepsilon_{v}$ associated with the counter-rotating system of Fig. 3 is estimater by assuming that the vortices are in solid-body rotation. Then $\varepsilon_{v}$ is taken as the product of the normal velocity $v$ and a length $l$ that varies from zero at the center of each vortex to the boundarylayer thickness at the outer edge, that is, at a radius of $.016 \mathrm{ft}$. Thus, with $\omega=160 \mathrm{rad} / \mathrm{sec}$

$\varepsilon_{v}=\frac{1}{.016} \int_{0}^{.016} \omega r \cdot l \mathrm{dr}=\frac{1}{.016} \int_{0}^{.016} \omega r^{2} \frac{\delta}{.016} \mathrm{dr}=0.84 \delta \mathrm{ft}^{2} / \mathrm{sec}$

For comparison with the turbulent boundary layer, Clauser ${ }^{10}$ found the following formula valid over a wide range of pressure gradients $\varepsilon_{t}=.018 U_{\infty} \delta^{*}$ where $U_{\infty}$ is the freestream velocity and $\delta^{*}$ is the displacement thickness of the boundary layer. For the results of Fig. 3, $U_{\infty}=110 \mathrm{fps}$ and if we take $\delta=10 \delta^{*}$ we get $\varepsilon_{t}=.2 \delta \mathrm{ft}^{2} / \mathrm{sec}$ for the turbulent boundary layer. Comparison of the magnitudes of $\varepsilon_{t}$ and $\varepsilon_{v}$ indicates that the eddy viscosity associated with the streamwise vortex system is of the order of four times that in the turbulent boundary layer, which is, in turn, of the same order as that in the wake. Since the rate of spread of a wake is roughly proportional to the square root of the eddy viscosity the effect of the streamwise vortex system would be to add substantially to the rate of dissipation of gradients in the flow. The generation of such a system near the hub for instance would tend to counteract the formation of large-scale circulations often found in turbomachines. ${ }^{7,8}$

The effect of vortex generators on the formation of the Kármán vortex street was investigated by means of schlieren flash photographs at subsonic speeds in an $8 \mathrm{in} . \times 13$ in. channel. Some of the photographs are shown in Fig. 5. A flat plate, $\frac{1}{2}$ in. thick, just behind the rounded leading edge, and tapering to $\frac{1}{4}$ in. at the trailing edge, spanned the 13 -in. height of the channel. The vortices were made visible to the schlieren setup by means of a heated wire just downstream of the trailing edge; the duration of the flashes was about $1 \mu \mathrm{sec}$. Details of the various configurations are given in the figure caption.

The photographs show that the generators exhibit a marked tendency to suppress the Kármán vortex street. In c), for the high turbulence condition, the vortices appear to dissipate more rapidly than in b); in d), taken with horizontal knife edge the streamwise vortices are evident behind the generators but the Kármán vortices show faintly in the lower half as well. In e) the suppression is evident for the thickened boundary layer resulting from roughness at the leading edge. In f) the suppression is shown for a lifting surface.

In other tests, when the generators were mounted near the leading edge (at .3 chord) they had no discernible suppression effect, and when the generators were mounted on only one surface the suppression was much reduced. A photograph taken at an indicated freestream Mach number of 0.6 , which was near the choking Mach number, showed the suppression effect to exist at high speeds as well. 


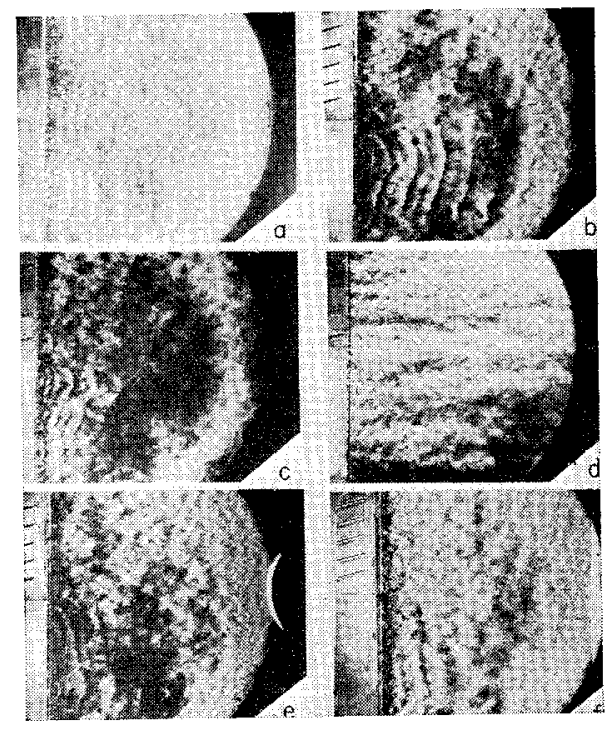

Fig. 5 Schlieren flash photographs of wake behind flat plate; heated wire behind $\frac{1}{4}$-in.-thick trailing edge; a) clean surface, $V=265 \mathrm{fps}$; b) and e) with vortex generators .05 in. high on upper halves of both surfaces, $V=265 \mathrm{fps}$; c) (vertical knife edge) and d) (horizontal knife edge) with $\frac{1}{4}$-in. rod $\frac{1}{2}$ in. upstream of leading edge; e) with 390 micron beads covering leading $10 \%$ of plate; $f$ ) with vortex generators .08 in. high on both surfaces, $5^{\circ}$ angle of attack, $V=170 \mathrm{fps}$.

The Strouhal number $S=f d / V$, where $f$ is the frequency, $d$ the effective width of the body, and $V$ is the velocity, is constant for a given body over a wide range of velocities. For the photographs of Fig. 5, the Strouhal number becomes simply the ratio of the trailing edge thickness to the spacing of the vortices. The value is about 0.25 , in satisfactory agreement with observations by many experimenters.

The details of the mechanism by which the vortex street is suppressed are not clear. Apparently the spanwise periodic flow associated with the streamwise vortex system at the trailing edge is intense enough to counteract the essentially twodimensional flow instability that leads to the formation of the Kármán vortex street.

Recent investigations directed toward finding the secrets of ultraquiet flight ${ }^{11-13}$ have concentrated on the mechanisms developed by owls. Kroeger, Gruschka, Helvey et al. ${ }^{11}$ found that the noise energy is concentrated largely in the low frequency bands, presumably because of a damping effect on the higher frequencies of leading edge serrations and soft wing plumage.

Hersh and Hayden ${ }^{12}$ following initial work by Soderman, ${ }^{13}$ concentrated on the function of the comblike structure at the leading edges of the owl's wings. They found that serrated comblike projections properly located near the leading edges of airfoils and fan blades suppressed the sound sources generated by the Kármán vortex street over the Reynolds number range $\left(8.3 \times 10^{4}\right.$ to $\left.3.33 \times 10^{5}\right)$ corresponding to predominantly laminar boundary layers. Oil film observations indicated that the serrations generated streamwise vortices on both surfaces. The authors conclude that these were responsible for the suppression of the vortex street, as well as for the virtual disappearance of the laminar separation bubble on the upper surface. They hypothesize that these two phenomena are coupled in the sense that the streamwise, or TaylorGoertler vortices trip the laminar layer thus eliminating the separation bubble and the periodic wake. The suppression was effective up to the Reynolds number at which the boundary layer on both surfaces was turbulent at the trailing edge.

Serrations near the leading edge are thus not effective in suppressing the vortex street shed from surfaces with turbulent boundary layers, such as those shown in Fig. 5. A reason may be that if streamwise vortices are generated in the laminar layer they become so dispersed by the high turbulence in the transition region that they are ineffective in suppressing the formation of the vortex street. It appears then that the serrations used by Soderman et al. suppress the "laminar vortex street" while the inclined wavy surface of Fig. 5, and presumably 3 and 4 as well, suppress the "turbulent". These are distinguished from each other in that the laminar type is periodic while the turbulent type is almost periodic, approaching broad band. Both, however, exhibit high spanwise correlation.

Hersh and Hayden ${ }^{12}$ measured forces and mean velocity distributions in the wake with and without serrations. They find that $x / c=.08$ and .17 the wake width is decreased when serrations are added, and the force measurements indicate a 1 to $5 \%$ drag decrease. The locations of the wake traverses are almost exactly those for the measurements of Table 1 and Fig. 3. They do not mention a spanwise periodicity so it would appear that the vortices generated by the serrations are appreciably weaker than those generated by the surface waviness. The measured drag decrease indicates that the addition of the serrations causes a relatively large decrease in form drag of the airfoil in the laminar flow regime; this decrease must be great enough to overcome the form drag of the serration surface and the increase in skin friction associated with the increased mixing due to the streamwise vortices. Momentum loss measurements at $x / c=4$ for the measurements reported here show about a $5 \%$ increase in drag in the turbulent flow regime for the configuration corresponding to Fig. 3 . It is likely, however, that the drag increment would be less with vortex generators on the lower rather than on upper surface only; at any rate a much more detailed investigation would be required to determine a configuration that gives the maximum suppression and wake distortion with minimum drag penalty.

\section{Conclusions}

Exploratory measurements of vortex shedding by a bluff body and of wake characteristics behind an airfoil indicate that relatively shallow inclined surface waviness near the trailing edge has the following effects: 1) The streamwise vortices generated induce a spanwise periodicity in the wake, with the result that a following blade experiences significant reductions in the unsteady forces and in the noise generation. 2) The Kármán vortex street is suppressed over a large Reynolds-number range including the completely turbulent regime. A result would be the attenuation of an important source of sound from aircraft in landing, fans, propellers, turbomachinery, etc.

\section{References}

${ }^{1}$ Kemp, N. H. and Sears, W. R., "Aerodynamic Interference due to Viscous Wakes in Turbomachines," Journal of the Aeronautical Sciences, Vol. 22, No. 7, 1955, pp. 478-483.

2 Kemp, N. H. and Sears, W. R., "Aerodynamic Interference between Moving Blade Rows," Journal of the Aeronautical Sciences, Vol. 20, No. 9, 1953, pp. 585-598.

${ }^{3}$ Kuethe, A. M., "Boundary Layer Control of Flow Separation and Heat Transfer," U.S. Patent No. 3,578,264, May 11, 1971. Patent applied for on further claims.

${ }^{4}$ Pearcey, H. H., "Boundary Layer Control for Aerofoils and Wings," Boundary Layer and Flow Control, Vol. 2, edited by G. V. Lachmann, Pergamon Press, New York, 1961, pp. 1260-1333.

${ }^{5}$ Rainbird, W. J., Crabbe, R. S., Peake, D. J., and Meyer, R. F., "Some Examples of Separation in Three-Dimensional Flows," Canadian Aeronautics and Space Journal, Vol. 12, No. 10, 1966, pp. 409-423.

6 Luce, Ross G., and Hoehne, V. O., "Comparison of the Boundary Layer Control Capabilities of Wave-Element and Vane Vortex Generators," Feb. 26, 1970, Battelle Development Corp. Rept. 
${ }^{7}$ Squire, H. B. and Winter, K. G., "The Secondary Flow in a Cascade of Airfoils in a Nonuniform Stream," Journal of the Aeronautical Sciences, Vol. 18, No. 4, 1951, p. 271.

${ }^{8}$ Hawthorne, W. R., "Some Aerodynamic Problems of Aircraft Engines," Journal of the Aeronautical Sciences, Vol. 24, No. 10, 1957, pp. 713-731.

${ }^{9}$ Rao, G. V. R., "Use of Leaning Vanes for Fan Noise Reduction," AIAA Paper 72-126, San Diego, Calif., 1972.

${ }^{10}$ Clauser, Francis, "The Turbulent Boundary Layer," Advances in Applied Mechanics, Vol. 4, Academic Press, 1956, pp. 1-51.
${ }^{11}$ Kroeger, R. A., Gruschka, H. D., Helvey, T. C. et al., "Low Speed Aerodynamics for Ultra-Quiet Flight," TR AFFDL-TR-7175, 1972, Air Force Flight Dynamics Lab., Wright-Patterson Air Force Base, Ohio.

${ }^{12}$ Hersh, A. S. and Hayden, R. E., "Aerodynamic Sound Radiation from Lifting Surfaces with and without Leading-Edge Serrations," NASA CR 114370, 1971.

${ }^{13}$ Soderman, P. T., "Effects of Leading-Edge Serrations on a Two-Dimensional Airfoil in the Ames 7- by 10-foot Wind Tunnel," TN, NASA (to be published).

\title{
Lifting Fan Noise Studies with Superimposed Cross Flows
}

\author{
G. KRISHNAPPA* \\ National Research Council of Canada, Ottawa, Ontario, Canada
}

\begin{abstract}
Experimental studies on noise radiation from a single-stage lifting fan operating at subsonic tip speeds are described. The effects on noise radiation of inlet flow distortion as a result of superimposed cross flow are presented. Under zero cross-flow conditions, narrow band analysis of the results shows the presence of discrete "shaft order tones," in addition to high-level tones at blade-passing frequencies at a subsonic tip speed of 628 fps. Propagating modes of these tones are identified with some reasonable success based on observed directivity patterns, using Tyler and Sofrin's radiation formula. Only the shaft orders at multiples of stator blade numbers giving a plane wave radiation are in evidence at lower fan speeds. Under cross-flow conditions, there is an increase of up to $10 \mathrm{db}$ in the level of broadband noise. The shaft order tones become less conspicuous because of this rise in broadband noise. The radiation patterns of blade-passing tones show strong dependence on the cross flow. An unexpected reduction in peak blade-passing tone levels at some conditions of cross flow suggests the two interaction mechanisms, inflow distortion with rotor and rotor with stator, are interdependent.
\end{abstract}

\section{Introduction}

A LIFTING fan in the form of the fan-in-wing configuration is one of the potential lift-producing systems for the takeoff and landing operations for VTOL aircraft. The fan noise problem in this type of installation is aggravated by three special factors. 1) The fan assembly is housed compactly inside the wing to yield good cruise performance. Consequently, there is a close spacing of the rotor and stator blade rows which increases the rotor-stator interaction noise. 2) Because of the shallow depth, there is a decided limit to the extent of attenuation that may be achieved by way of introduction of acoustic absorption material. 3) During the transition maneuvers from vertical to horizontal flight and vice versa, the forward velocity of the aircraft is superposed on the vertical downward flow into the fan. Previous experiments and theory have indicated substantial radial and circumferential flow variations in velocity during cross flow conditions at the entrance to a fan rotor installed in a shallow inlet. While these variations affect the aerodynamic performance of the fan, ${ }^{5}$ they are also likely to alter noise levels and radiating patterns by interacting with the rotor blades in a way similar to rotor-stator interaction.

The generally held concept of fan and compressor noise at subsonic speeds was that its spectrum was composed of discrete tones at blade-passing frequency and its harmonics,

Presented as Paper 72-128 at the AIAA 10th Aerospace Sciences Meeting, San Diego, Calif., January 17-19, 1972; submitted February 7, 1972; revision received June 23, 1972.

Index category: VTOL Propulsion.

* Research Officer, Engine Laboratory, Mechanical Engineering Division. superimposed on broadband noise extending over a wide frequency range. This concept was based on the experimental studies done at filter bandwidths higher than $6 \%$. The discrete tones were produced as a result of rotor-stator interaction, and the broadband noise was generated by the turbulence in the entering stream. The recent works of Mather, Savidge and Fisher ${ }^{1}$ and a few others have shown that the spectrum at narrow bandwidths reveals that what might have been regarded as broadband noise is comprised of several tones at integral shaft orders. The interaction of rotor blades which are slightly dissimilar as a result of geometrical bladeto-blade variations (spacing, incidence, twist, thickness, etc.) with the stator blades suggests acoustic pressure fields which repeat once per rotor revolution.

The distorted flow entering the inlet may interact with the rotor field in a similar way as the interaction of rotor blades with stators generating propagating modes. These modes radiate energy in a characteristic pattern which may be identified by using Tyler and Sofrin' ${ }^{2}$ analysis.

This paper presents some experimental work on the noise characteristics of a model-sized lifting fan, with and without cross flow over the wing. The experimental results at zero cross flow and cross-flow conditions are described in Secs. 4 and 5, with associated theoretical considerations in Sec. 2. The experimental results are discussed by identifying various propagating modes resulting from rotor-stator interactions and inlet flow-rotor interactions.

\section{Theoretical Considerations}

Tyler and Sofrin ${ }^{2}$ have shown the possibility of propagating pressure fields even at subsonic tip Mach numbers resulting 